\title{
Avaliação comparativa entre os coagulantes sulfato de alumínio ferroso e policloreto de alumínio para tratamento de água: estudo de viabilidade econômica
}

Comparative evaluation between ferrous aluminum sulfate and aluminum polychloride coagulants for water treatment: economic feasibility study

Evaluación comparativa entre sulfato de aluminio ferroso y coagulantes de policloruro de aluminio para el tratamiento del agua: estudio de viabilidad económica

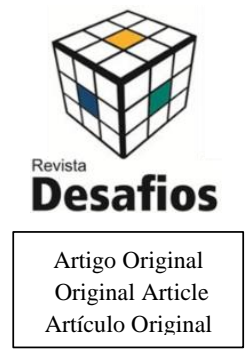

\author{
Karita Santos Lemos ${ }^{1}$, Silvio Quintino de Aguiar Filho ${ }^{1,2}$, Grasiele Soares Cavallini*1,2 \\ ${ }^{1}$ Curso de Química Ambiental, Universidade Federal do Tocantins, Gurupi, Brasil \\ ${ }^{2}$ Programa de Pós-Graduação em Química, Universidade Federal do Tocantins, Gurupi, Brasil
}

*Correspondência: Laboratório de Análises Ambientais, Universidade Federal do Tocantins,

Gurupi, Tocantins, Brasil, Rua Badejós, Zona Rural, CEP 77.402-970.

E-mail: grasiele@uft.edu.br

Artigo recebido em 26/10/2019 aprovado em 02/03/2020 publicado em 24/03/2020.

\section{RESUMO}

O presente trabalho compara o desempenho de dois coagulantes, o sulfato de alumínio ferroso (SAF) e o policloreto de alumínio (PAC) na remoção de turbidez para o tratamento de água para abastecimento público, utilizando como estudo de caso a represa Bananal que abastece o Município de Gurupi, TO. O estudo foi realizado em escala de bancada utilizando equipamento Jar test para os ensaios de coagulação, floculação e sedimentação, e para o ensaio de filtração foi utilizado filtro de areia própria para filtro de ETA. Para avaliar a eficiência do sistema foram testadas: diferentes dosagens dos coagulantes em distintas faixas de $\mathrm{pH}$, a velocidade de sedimentação e a taxa de filtração. O coagulante PAC, na dosagem de $12 \mathrm{mg} . \mathrm{L}^{-1}$, apresentou melhor desempenho na remoção de cor aparente e turbidez, com a menor concentração de ferro total e alumínio total residual. A dosagem de $15 \mathrm{mg} . \mathrm{L}^{-1}$ do coagulante SAF demonstrou um desempenho inferior, no entanto, ambos apresentaram resultados dentro dos limites estabelecidos pela Portaria MS no 2.914/2011. Desta forma, o estudo de custos indicou que economicamente a utilização do SAF é mais viável, embora resultados melhores tenham sido obtidos com PAC.

Palavras-chave: Filtração direta, PAC, SAF.

\section{ABSTRACT}

The present work compares the performance of two coagulants, ferrous aluminum sulphate (FAS) and aluminum polychloride (PAC) in the recovery of turbidity for the treatment of water for public supply, using as a case study in case of representations Gurupi, TO. The study was carried out on a bench scale using Jar test equipment for the coagulation, flocculation and sedimentation tests, and for the filtration test, a sand filter for the WTP filter was used. To evaluate the performance of the system, different doses of coagulants in different $p H$ ranges, sedimentation speed and filtration rate were tested. The PAC coagulant, at a dosage of $12 \mathrm{mg} . \mathrm{L}^{-1}$, shows better performance in removing apparent color and turbidity, with the lowest concentration of total iron and total residual aluminum. A dosage of $15 \mathrm{mg} . \mathrm{L}^{-1}$ of the coagulant FAS demonstrated inferior performance, however, both results were within the limits established by Portaria MS $n^{\circ} 2.914$ / 2011. Thus, the cost study indicates that the economic use of FAS is more feasible, although the best results have been used in the PAC.

Keywords: Direct filtration, PAC, FAS.

\section{RESUMEN}


El presente trabajo compara el desempeño de dos coagulantes, el sulfato de aluminio ferroso (SAF) y el policloruro de aluminio (PAC) en la eliminación de la turbidez para el tratamiento del agua para el suministro público, utilizando la presa de Bananal que suministra Municipio de Gurupi, TO. El estudio se llevó a cabo a escala de banco utilizando equipos de prueba Jar para las pruebas de coagulación, floculación y sedimentación, y para la prueba de filtración, se utilizó un filtro de arena para el filtro ETA. Para evaluar la eficiencia del sistema se probaron: diferentes dosis de coagulantes en diferentes rangos de $\mathrm{pH}$, la velocidad de sedimentación y la velocidad de filtración. El coagulante PAC, a una dosis de $12 \mathrm{mg} . \mathrm{L}^{-1}$, mostró un mejor rendimiento en la eliminación del color aparente y la turbidez, con la concentración más baja de hierro total y aluminio residual total. La dosis de $15 \mathrm{mg} . \mathrm{L}^{-}$ ${ }^{1}$ del coagulante SAF mostró un rendimiento inferior, sin embargo, ambos presentaron resultados dentro de los límites establecidos por la Ordenanza MS n $n^{o} 2.914$ / 2011. Por lo tanto, el estudio de costos indicó que el uso de SAF es más viable económicamente, aunque se han obtenido mejores resultados con PAC.

Descriptores: filtración directa, PAC, SAF.

\section{INTRODUÇÃO}

O acesso da população à água potável é vital para sua sobrevivência, o que torna os processos de tratamento de água essenciais para a saúde pública e o desenvolvimento urbano. As águas superficiais possuem diversos tipos de impurezas: gases dissolvidos, compostos orgânicos e inorgânicos dissolvidos e em suspensão, assim como, microrganismos (bactérias, algas e fungos) e coloides. Devido a esta diversidade, se fazem necessárias diferentes formas de tratamento para uma remoção efetiva dos contaminantes e o estabelecimento da potabilidade da água, os quais devem ser propostos de acordo com as características físico-químicas da água (PAVANELLI, 2001; DI BERNARDO et al., 2017).

Dentre as características físicas da água, a cor (verdadeira e aparente) e a turbidez se evidenciam para a avaliação da eficiência de sistemas de tratamento de água. A turbidez, segundo Richter (2009), é uma característica ótica da água que promove a dispersão e a absorção de um feixe de luz, devido ao desvio provocado pela presença de frações em suspensão e coloides. A coloração da água, segundo o mesmo autor, decorre de sua eficiência de absorção de certas radiações do espectro visível (450nm ou 550nm), e é devida, geralmente, às substâncias de origem mineral e orgânicas dissolvidas (cor verdadeira), no estado coloidal ou em suspensão (cor aparente).
As principais propriedades químicas da água mensuradas periodicamente em Estações de Tratamento de Água (ETA) são: alcalinidade e pH, pois ambas influenciam de forma relevante no processo de coagulação de águas de abastecimento. As interações entre partículas coloidais regem a capacidade de afastamento ou agregação das partículas, fundamentando a etapa de coagulação e floculação no tratamento de água. As forças externas correspondentes ao campo da gravidade ou ao cisalhamento também promovem a interação e as colisões entre partículas (PAVANELLI, 2001; DI BERNARDO et al., 2017).

Nas dispersões coloidais aquosas podem ocorrer: interação repulsiva de duplas camadas de cargas, forças de Van der Walls interação estérica repulsiva de cadeias de polímeros adsorvidos nas partículas, interação atrativa e interação hidrofóbica (JAFELICCI e VARANDA, 1999; RAMOS et al., 2018). Sendo assim, os mecanismos integrantes na coagulação de sistemas coloidais são complexos e abrangem propriedades de superfície tais como: potenciais elétricos, interações solvente-soluto e solvente-partículas, produtos de solubilidade, condições de mistura e de $\mathrm{pH}$, entre outros (GONÇALVES et al., 2006), as quais são responsáveis pelos mecanismos de: compressão de camada difusa, adsorção e neutralização, varredura e formação de 
pontes durante o processo de coagulação (DI BERNARDO et al., 2017).

\section{Características dos coagulantes em estudo}

O Policloreto de alumínio (PAC) é um coagulante inorgânico polimerizado catiônico de baixo peso molecular e pré-polimerizado, apresentando-se na forma líquida com aparência viscosa. O mesmo possui cadeias de polímeros préformadas, contendo uma alta concentração de carga catiônica na unidade polimérica. Apresenta capacidade de formar flocos com uma velocidade superior aos coagulantes tradicionais, não prépolimerizados, o que garante que os flocos possuam maior densidade, e assim, a precipitação ocorre mais rapidamente (CARDOSO, 2006).

O PAC geralmente é formulado como: $\mathrm{A} \ln (\mathrm{OH}) \mathrm{mCl}_{3} \mathrm{n}-\mathrm{m}, \quad$ com adição de pequenas quantidades de outros compostos. A basicidade do produto é representada pela relação $\mathrm{m} / 3 \mathrm{n}$, com essa característica o mesmo libera no processo de hidrólise menor quantidade de ácido do que a liberada pelo Sulfato de alumínio ferroso (SAF). Consequentemente, ocorre uma menor variação do $\mathrm{pH}$ do efluente tratado, reduzindo a quantidade de neutralizante, caso haja necessidade de correção de $\mathrm{pH}$ (CARDOSO, 2006).

$\mathrm{O}$ sulfato de alumínio $\left(\mathrm{Al}_{2}\left(\mathrm{SO}_{4}\right)_{3} \cdot 18 \mathrm{H}_{2} \mathrm{O}\right)$ é considerado o coagulante químico mais usado no Brasil no tratamento de água de abastecimento público, em razão da alta eficiência na remoção de sólidos em suspensão e pelo relativamente baixo custo para sua aquisição. A Reação 1 demonstra o produto formado quando o sulfato de alumínio é adicionado na água em condições alcalinas (ECKENFELDER,1989):

$\mathrm{Al}_{2}\left(\mathrm{SO}_{4}\right)_{3}+18 \mathrm{H}_{2} \mathrm{O}+3 \mathrm{Ca}(\mathrm{OH})_{2} \rightarrow 3 \mathrm{CaSO}_{4} 2 \mathrm{Al}(\mathrm{OH})_{3}+18 \mathrm{H}_{2} \mathrm{O}$

(Reação 1)
De acordo com Okuda et al. (2014), altas concentrações de alumínio no meio podem ocasionar problemas à saúde humana, inclusive aceleram o processo degenerativo do Mal de Alzheimer, por isso deve haver cautela nas suas dosagens. Além do mais, a utilização de sais de alumínio oportuniza consumo da alcalinidade da água em tratamento, acrescentando custos adicionais com produtos químicos utilizados na correção do seu pH.

Para valores elevados da dosagem de sulfato de alumínio (comumente superiores a $30 \mathrm{mg} . \mathrm{L}^{-1}$ ) e de pH em torno de 6 e 8, há formação do precipitado $\mathrm{Al}(\mathrm{OH})_{3}$, porém, quando o $\mathrm{pH}$ é inferior a cerca de 5,7, pode haver formação e predominação de espécies poliméricas do tipo $\mathrm{Al}_{13} \mathrm{O}_{4}(\mathrm{OH})_{27}{ }^{4+}$ (Reação 2).

$13 \mathrm{Al}^{3+}+28 \mathrm{H}_{2} \mathrm{O} \rightarrow \mathrm{Al}_{13} \mathrm{O}_{4}(\mathrm{OH})_{24}{ }^{7+}+32 \mathrm{H}^{+}(-\log \mathrm{K}=97)$ (Reação 2)

Quando o sulfato de alumínio é acrescido à água, ocorrem reações de hidrólise, intermediárias, que antecedem a formação do precipitado amorfo. Essas reações liberam prótons $\left(\mathrm{H}_{3} \mathrm{O}^{+}\right)$, que consomem substâncias que conferem alcalinidade natural à água, da ordem de $1 \mathrm{mg} . \mathrm{L}^{-1}$ de sulfato de alumínio $\left[\mathrm{Al}_{2}\left(\mathrm{SO}_{4}\right)_{3} \cdot 14,3 \mathrm{H}_{2} \mathrm{O}\right]$ para cada $0,5 \mathrm{mg} \cdot \mathrm{L}^{-1}$ de $\mathrm{CaCO}_{3}$ (DI BERNARDO, 2005), na ausência de substâncias tamponantes como o carbonato de cálcio, o pH do meio pode cair drasticamente.

O minério para obtenção de muitos compostos de alumínio é a bauxita, a qual apresenta óxidos de ferro (hematita, magnetita e goetita, entre outros) como uma de suas impurezas, causando alterações no aspecto físico do minério, podendo apresentar-se como um sólido marrom-escuro ferruginoso (CONSTANTINO, et al. 2002). Para reduzir os custos da produção do coagulante o sulfato de alumínio pode ser comercializado como Sulfato de alumínio ferroso, logo que, íons ferro também tem ação coagulante. A 
formação de hidróxido férrico insolúvel é produzida numa faixa de $\mathrm{pH}$ 3,0 - 13,0, como observado na seguinte reação 3 (ECKENFELDER, 1989):

$$
\mathrm{Fe}_{3}{ }^{+}+3 \mathrm{OH}^{-} \rightarrow \mathrm{Fe}(\mathrm{OH})_{3} \quad \text { (Reação 3) }
$$

A Tabela 1 apresenta as características dos coagulantes PAC e SAF, conforme o fabricante.

Tabela 1 - Ficha técnica dos coagulantes PAC e SAF.

\begin{tabular}{l|c|c|l}
\hline \multicolumn{1}{c|}{ Parâmetros } & PAC & SAF & Especificação \\
\hline $\begin{array}{l}\text { Óxido de } \\
\text { alumínio } \\
\left(\mathrm{Al}_{2} \mathrm{O}_{3}\right) \%\end{array}$ & $10-14$ & 7,7000 & mín: 7,000 \\
$\begin{array}{l}\text { Óxido de ferro } \\
\left(\mathrm{Fe}_{2} \mathrm{O}_{3}\right) \%\end{array}$ & 1,1500 & 1,1500 & máx: 1,400 \\
$\begin{array}{l}\text { Densidade } \\
\left(\mathrm{g} \cdot \mathrm{cm}^{-3}\right)\end{array}$ & 1,3410 & 1,3410 & $\begin{array}{l}\text { mín: } 1,335- \\
\text { máx: } 1,345\end{array}$ \\
$\begin{array}{l}\text { Acidez } \\
\begin{array}{l}\text { Basicidade livre } \\
\left(\mathrm{Al}_{2} \mathrm{O}_{3}\right)\end{array}\end{array}$ & 0,0400 & 0,0400 & máx: 0,200 \\
\hline
\end{tabular}

Fonte: SULFAGO®, 2018.

Embora tanto o PAC quanto o SAF apresentem eficiência consolidada para o tratamento de água, estudos para a definição do coagulante mais adequado são necessários para se obter a otimização operacional do sistema. Neste sentido, este estudo tem por finalidade avaliar a viabilidade dos coagulantes PAC e SAF para aplicação na ETA de Gurupi, Tocantins, considerando os fatores eficiência de tratamento e custo operacional.

\section{MATERIAIS E MÉTODOS}

O estudo foi realizado na Represa Bananal pertencente ao município de Gurupi-TO, formada a partir das águas do Córrego Bananal, construída pela Companhia de Saneamento do Tocantins, em agosto de 1995, e atualmente gerida pela empresa BRK Ambiental. A mesma apresenta área de aproximadamente 260 hectares, volume acumulado de $12.998 .869,15 \mathrm{~m}^{3}$ e média de $5 \mathrm{~m}$ de profundidade. $\mathrm{O}$ município de Gurupi está localizado na região sul do Estado do Tocantins e corresponde ao $3^{\circ}$ maior município do Estado em número de habitantes, ultrapassando 80 mil (PREFEITURA DE GURUPI, 2017).

As amostras de água foram coletadas na ETA Gurupi, na chegada da água bruta à estação. A água foi armazenada em galões com capacidade de 20 litros previamente limpos e utilizada logo após a coleta, foram realizadas um total de três coletas, distribuídas nos meses de abril e maio de 2018. Primeiramente, realizou-se ensaios de coagulação / floculação / sedimentação avaliando os coagulantes sulfato de alumínio ferroso (SAF) e o policloreto de alumínio (PAC) para o estabelecimento de dosagens ótimas dos coagulantes, na segunda etapa do estudo foram realizados ensaios de coagulação/floculação/filtração direta, a partir dos resultados da primeira etapa.

Os ensaios de coagulação / floculação / sedimentação foram realizados em equipamento Jar test, com gradiente de mistura rápida (coagulação) de $500 \mathrm{~s}^{-1}$ (270 RPM) por 1 minuto, gradiente de mistura lenta (floculação) de $65 \mathrm{~s}^{-1}$ (70 RPM) por 10 minutos e velocidades de sedimentação de 1,$34 ; 0,67 ; 0,45$ e $0,33 \mathrm{~cm} \cdot \mathrm{min}^{-1}$. As configurações avaliadas são descritas na Tabela 2. O pH foi ajustado com soluções de ácido sulfúrico 0,1 mol.L $\mathrm{L}^{-1}$ e hidróxido de sódio 0,1 mol.L $L^{-1}$. A eficiência dos ensaios foi avaliada pela remoção de turbidez em um turbidímetro portátil da marca Hach.

Tabela 2 - Dosagens e valores de $\mathrm{pH}$ avaliados nos ensaios de coagulação e sedimentação com PAC e SAF.

\begin{tabular}{c|c|l}
\hline Coagulante & $\begin{array}{c}\text { Dosagem } \\
\left(\text { mg.L }^{-1}\right)\end{array}$ & \multicolumn{1}{c}{$\mathbf{p H}$} \\
\hline \multirow{2}{*}{ PAC } & 5 & Natural $(6,7)$ \\
\cline { 2 - 3 } & 10 & $\begin{array}{l}\text { Natural }(6,7) ; 5,5 ; 6,0 ; \\
6,5 ; 7,0 ; 7,5 \text { e } 8,0\end{array}$ \\
\hline
\end{tabular}




\begin{tabular}{c|c|l}
\hline & 15 & $\begin{array}{l}\text { Natural }(6,7) ; 5,5 ; 6,0 ; \\
6,5 ; 7,0 ; 7,5 \text { e } 8,0\end{array}$ \\
\hline \multirow{4}{*}{ SAF } & 5 & Natural $(6,7)$ \\
\cline { 2 - 3 } & 10 & $\begin{array}{l}\text { Natural }(6,7) ; 5,5 ; 6,0 ; \\
6,5 ; 7,0 ; 7,5 \text { e } 8,0\end{array}$ \\
\cline { 2 - 3 } & 15 & $\begin{array}{l}\text { Natural }(6,7) ; 5,5 ; 6,0 ; \\
6,5 ; 7,0 ; 7,5 \text { e } 8,0\end{array}$ \\
\hline
\end{tabular}

Os ensaios de coagulação/floculação/filtração direta foram realizados primeiramente em equipamento Jar test, com o gradiente de mistura rápida de $500 \mathrm{~s}^{-1}$ (270 RPM) por 1 minuto em $\mathrm{pH}$ natural, gradiente de mistura lenta de $65 \mathrm{~s}^{-1}$ (70 RPM) por 10 minutos, conforme a Tabela 3. Para as dosagens com os melhores resultados de turbidez foram avaliados os seguintes parâmetros: cor aparente, alumínio, ferro, condutividade, sólidos dissolvidos totais (SDT) e $\mathrm{pH}$, de acordo com a metodologia estabelecida pelo Standard Methods for the Examination of Water and Wastewater (APHA, 1999).

Tabela 3 - Dosagens de PAC e SAF e o volume filtrado.

\begin{tabular}{|c|c|c|c|}
\hline Coagulante & $\begin{array}{c}\text { Dosagem } \\
\left(\mathrm{mg.L}^{-1}\right)\end{array}$ & pH & $\begin{array}{c}\text { Volume } \\
\text { filtrado } \\
\text { (L) }\end{array}$ \\
\hline \multirow{3}{*}{ PAC } & 8 & \multirow{4}{*}{$\begin{array}{c}\text { Natural } \\
(6,7)\end{array}$} & 2 \\
\hline & 10 & & 6 \\
\hline & 12 & & 6 \\
\hline SAF & 15 & & 2 \\
\hline
\end{tabular}

Após a mistura lenta a água foi submetida à filtração em um filtro de areia com área superficial de $9,42 \mathrm{~cm}^{2}$ e camada de areia de $10 \mathrm{~cm}$ de altura (Figura 1a e b). A areia foi fornecida pela BRK e tem destinação própria para filtro de água. As taxas de filtração (Vazão/área superficial do filtro) variaram de 22 a $38 \mathrm{~m}^{3} \mathrm{~m}^{-2} \mathrm{~h}^{-1}$.
Figura 1 - a) Filtro de areia em escala laboratorial. b) Parte inferior do filtro.
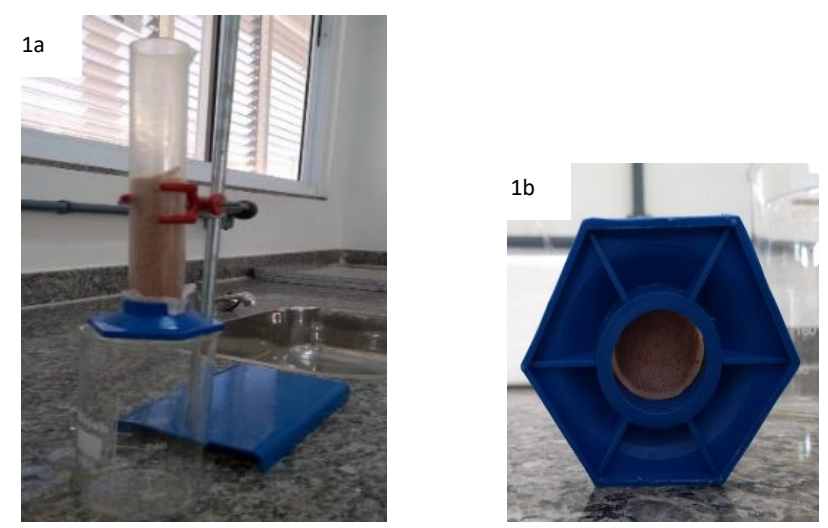

\section{RESULTADOS E DISCUSSÃO}

No primeiro ensaio de coagulação foram avaliadas as dosagens de 5,10 e $15 \mathrm{mg} . \mathrm{L}^{-1}$ para cada coagulante, no $\mathrm{pH}$ natural da água bruta, o qual foi de 6,7. Os resultados são demonstrados na Figura 2, identificando as remoções mais significativas no ensaio.

Figura 2 - Resultados do ensaio de coagulação da água bruta em $\mathrm{pH}$ natural $(\mathrm{pH}=6,7)$ com diferentes dosagens dos coagulantes SAF e PAC.

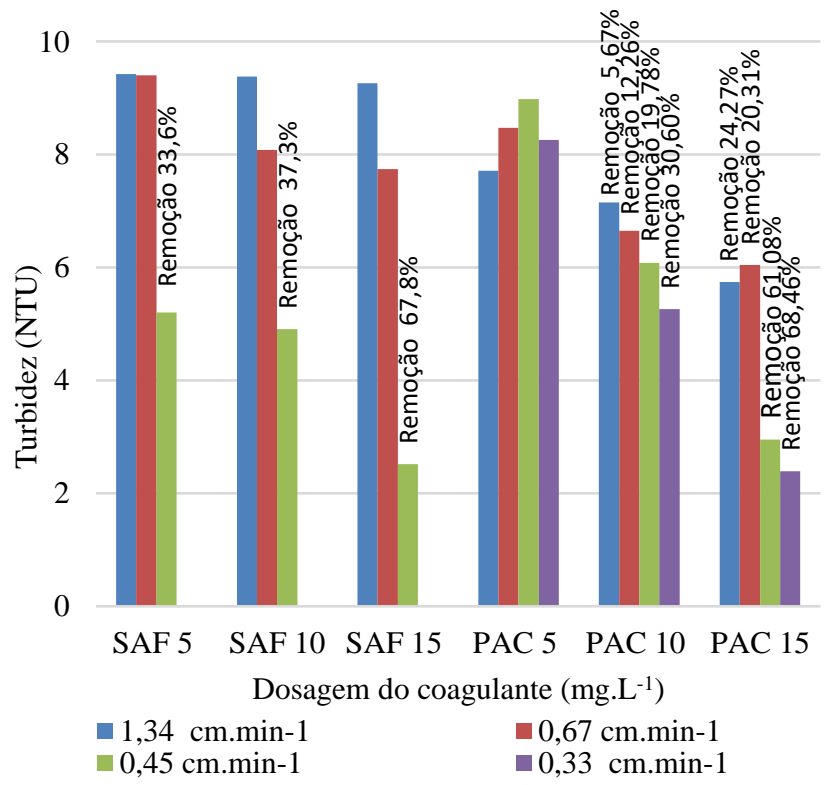

De acordo com os resultados apresentados na Figura 2, para ambos os coagulantes, a dosagem de 15 
mg.L $L^{-1}$ demostrou as melhores eficiências de remoção de turbidez, alcançando remoção superior a $60 \%$. Mesmo com o aumento do tempo de sedimentação (velocidade de sedimentação de $0,45 \mathrm{~cm} \cdot \mathrm{min}^{-1}$ ), a remoção se manteve próxima a $60 \%$.

Para avaliar a influência do $\mathrm{pH}$ na ação coagulante foram avaliadas duas concentrações, a de $15 \mathrm{mg} . \mathrm{L}^{-1}$ por ter apresentado melhor desempenho no ensaio anterior, e também a dosagem de $10 \mathrm{mg} . \mathrm{L}^{-1}$, logo que é esperado um melhor desempenho dos coagulantes quando o pH ótimo de coagulação é utilizado.

Os resultados dos ensaios utilizando $10 \mathrm{mg} . \mathrm{L}^{-}$ ${ }^{1}$ dos coagulantes são apresentados nas Figura 3 e 4, com identificação das melhores eficiências de remoção obtidas.

Figura 3 - Resultados do ensaio de coagulação da água bruta com variação de $\mathrm{pH}$ e dosagem fixa de 10 $\mathrm{mg} . \mathrm{L}^{-1}$ do coagulante SAF.

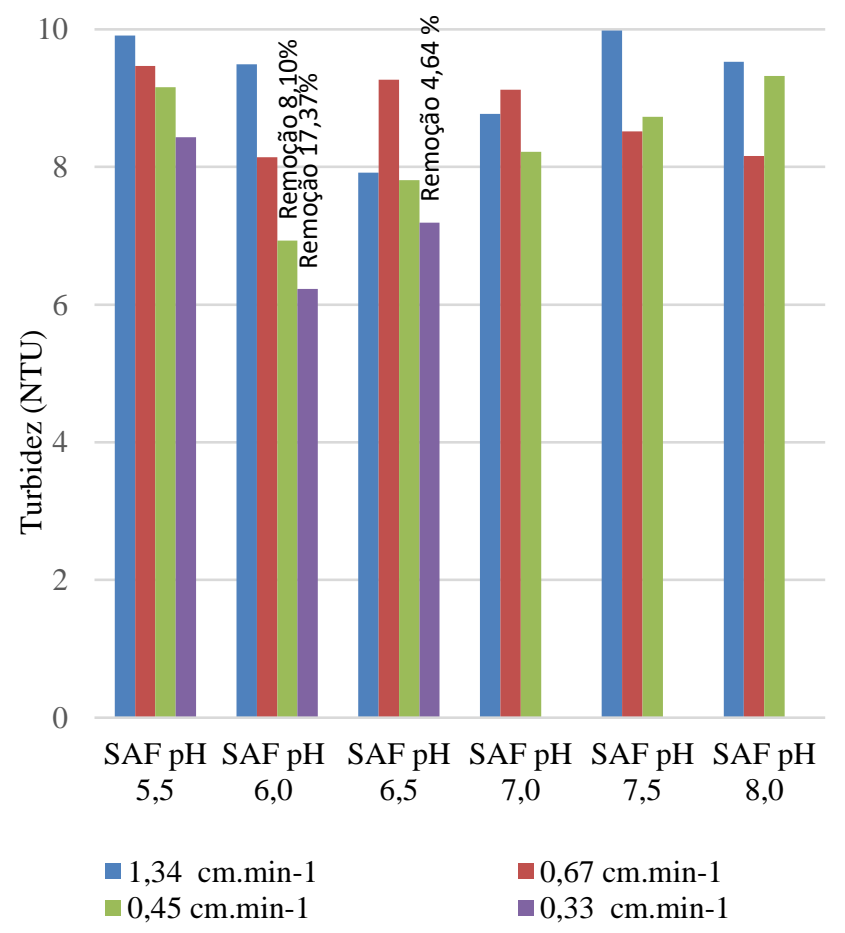

Figura 4 - Resultados do ensaio de coagulação da água bruta com variação de $\mathrm{pH}$ e dosagem fixa de $10 \mathrm{mg} . \mathrm{L}^{-}$ ${ }^{1}$ do coagulante PAC.

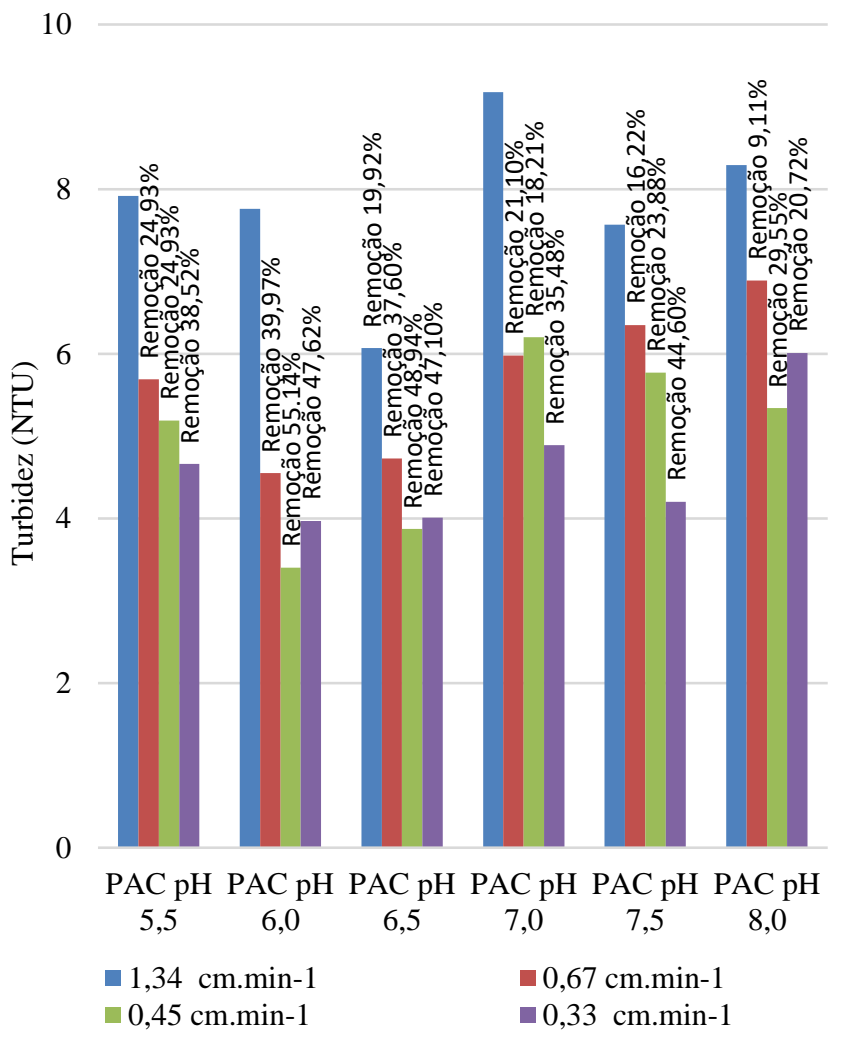

De acordo com a Figura 3, a porcentagem de remoção de turbidez com a dosagem de $10 \mathrm{mg} . \mathrm{L}^{-1}$ do SAF não demonstrou resultados significativamente eficientes, mesmo com a variação de $\mathrm{pH}$, pois a remoção não atingiu $20 \%$.

Para o ensaio com PAC a dosagem de 10 mg. $L^{-1}$ demonstrou maior eficiência de remoção de turbidez nos valores de $\mathrm{pH}$ 6,0 e de 6,5, atingindo 50\% de remoção em velocidade de sedimentação 0,45 cm.min ${ }^{-1}$ e pH 6,0, mantendo-se estável mesmo em velocidade de sedimentação de $0,33 \mathrm{~cm} \cdot \mathrm{min}^{-1}$.

Os resultados dos ensaios utilizando $15 \mathrm{mg} . \mathrm{L}^{-}$

${ }^{1}$ dos coagulantes são apresentados nas Figura 5 e 6. 
Figura 5 - Resultados do ensaio de coagulação da água bruta com variação de $\mathrm{pH}$ e dosagem fixa de $15 \mathrm{mg} . \mathrm{L}$

${ }^{1}$ do coagulante SAF.

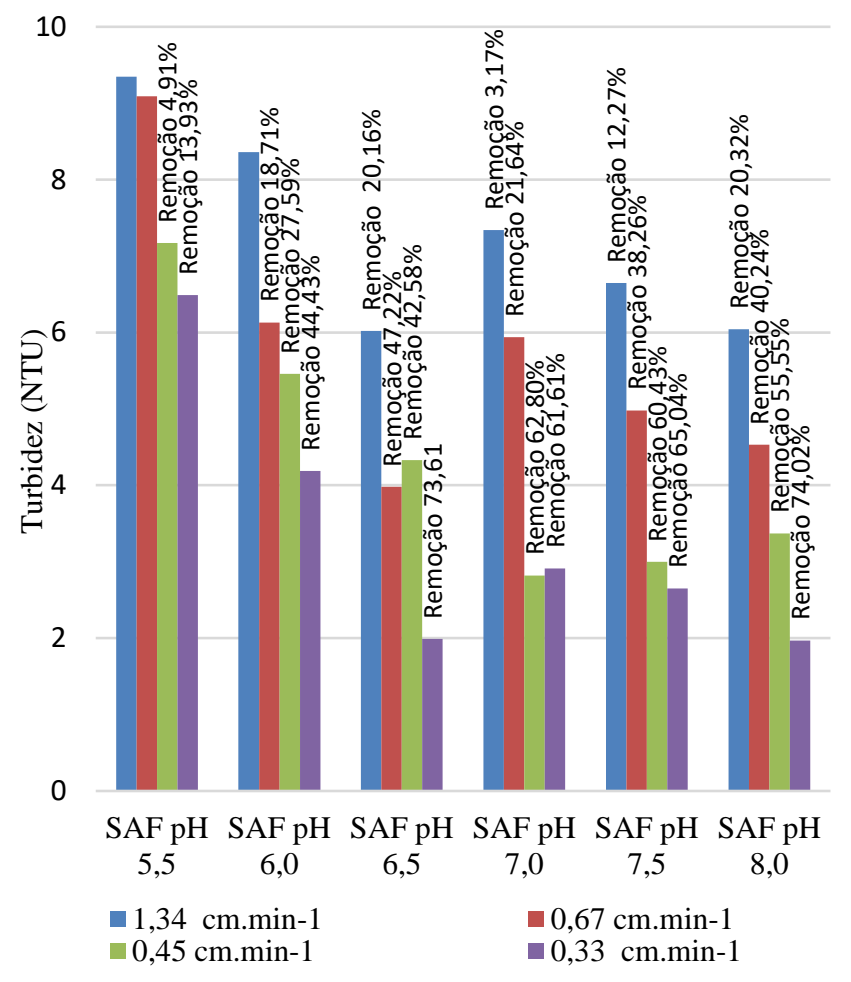

Figura 6 - Resultados do ensaio de coagulação da água bruta com variação de $\mathrm{pH}$ e dosagem fixa de $15 \mathrm{mg}$. $\mathrm{L}^{-}$ ${ }^{1}$ do coagulante PAC.

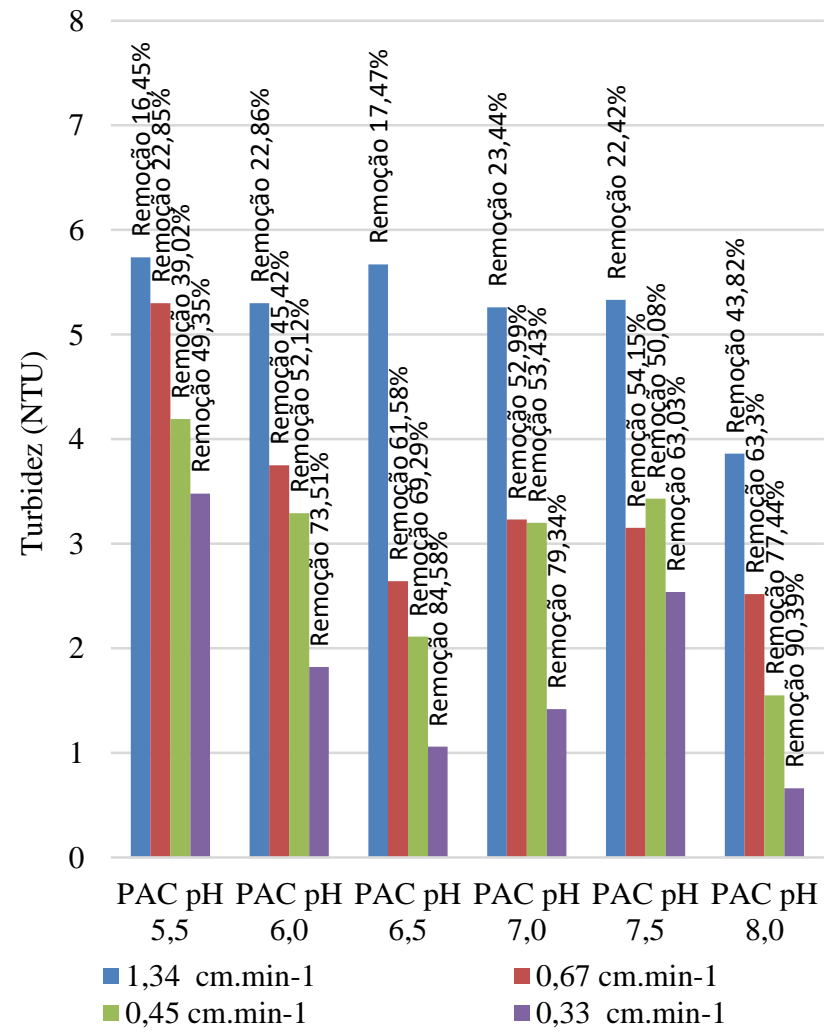

Para o coagulante SAF (15 mg. $\left.\mathrm{L}^{-1}\right)$, nos valores de $\mathrm{pH}$ entre 6,5 e 8,0 foram observadas maiores eficiências de remoção de turbidez, atingindo valores próximos a 70\% de remoção em velocidade de sedimentação de $0,33 \mathrm{~cm} \cdot \mathrm{min}^{-1}$.

Para a dosagem de $15 \mathrm{mg} . \mathrm{L}^{-1}$ de PAC, as melhores eficiências de remoção de turbidez apresentaram-se em pH 6,5 e 8,0. O tempo de sedimentação foi bastante significativo neste ensaio, e na velocidade de sedimentação de $0,33 \mathrm{~cm} \cdot \mathrm{min}^{-1}$ obteve-se a uma remoção de 90,39\% em pH 8,0. De acordo com Constantino (2002), o forte poder de coagulação mediante a formação rápida dos flocos torna a ação coagulante do PAC mais satisfatória, formando flocos maiores e mais uniformes, aumentando a velocidade de decantação, a exemplo disso, na velocidade de sedimentação $0,45 \mathrm{~cm} \cdot \mathrm{min}^{-1}$ com PAC foram obtidos eficiências de remoção de turbidez próximas ao ensaio com SAF na mesma dosagem e velocidade de sedimentação de 0,33 cm.min ${ }^{-1}$.

A partir dos estudos de variação de $\mathrm{pH}$ da amostra, constatou-se que para ambos os coagulantes, as melhores eficiências de remoção de turbidez foram obtidas próximas do $\mathrm{pH}$ natural da amostra $(6,7)$, demonstrando que a correção de pH não é justificável para esta amostra.

Com o auxílio destes resultados e visando uma redução na quantidade de coagulante utilizado foram realizados ensaios de coagulação seguida de filtração, simulando uma filtração direta, na qual a etapa de floculação ocorre durante a passagem pelo filtro.

Os ensaios de coagulação seguidos de filtração foram realizados com as dosagens de $8 \mathrm{mg} . \mathrm{L}^{-1}, 10$ mg.L $L^{-1}$ e $12 \mathrm{mg} . \mathrm{L}^{-1}$ para o PAC e $15 \mathrm{mg} . \mathrm{L}^{-1}$ para o SAF em $\mathrm{pH}$ natural. Neste ensaio o volume filtrado para 
cada dosagem foi de 2 litros, sendo o filtro lavado a cada nova dosagem. Os resultados são apresentados na Figura 7, a qual apresenta o volume filtrado em função do tempo, e também apresenta as porcentagens de remoção de turbidez ao longo da filtração.

Figura 7 - Filtração com dosagens de 8 mg.L $L^{-1}, 10$ mg. $L^{-1}$ e $12 \mathrm{mg} . \mathrm{L}^{-1}$ para o PAC e $15 \mathrm{mg} . \mathrm{L}^{-1}$ para o SAF, todos em $\mathrm{pH}$ natural.

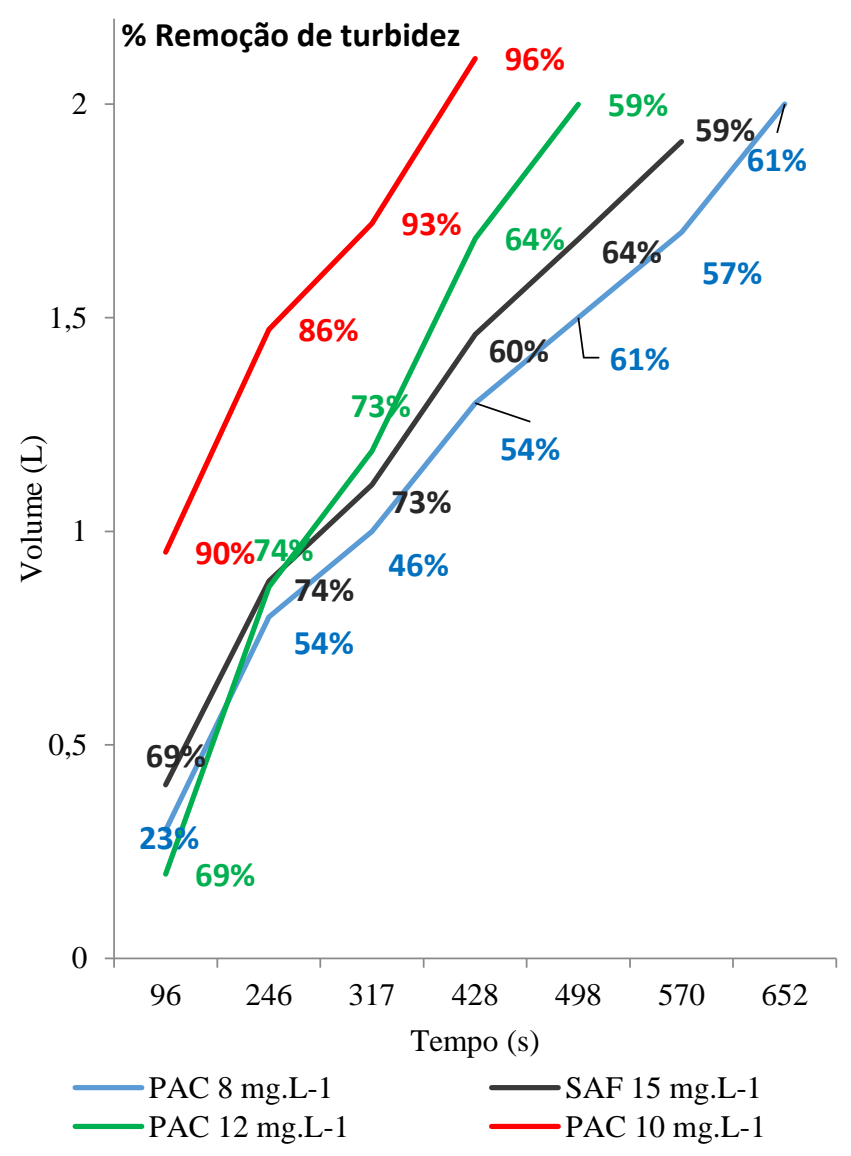

Para o coagulante SAF com dosagem de 15 mg. $L^{-1}$ houve uma remoção máxima de turbidez semelhante a do PAC (12 mg.L $\left.\mathrm{L}^{-1}\right)$, atingindo $74 \%$, porém com um taxa de filtração $24 \mathrm{~m}^{3} \cdot \mathrm{m}^{-2} \cdot \mathrm{h}^{-1}$.

A linearidade de curva é descrita pelo $\mathrm{R}^{2} \mathrm{e}$ indicam possíveis alterações na taxa de filtração (Vazão em função da área superficial do filtro), sendo que, quanto mais próximo de 1 , menor a alteração da taxa. A taxa de filtração constante indica que não houve entupimento do filtro ao longo da filtração, enquanto que alterações bruscas na taxa de filtração indicariam que o filtro deveria ser lavado devido a

perda de carga hidráulica, podendo também haver transpasse de sólidos para a água filtrada.

Para todas as dosagens avaliadas as taxas de filtração se mantiveram lineares e declinantes, demonstrando a diminuição da porosidade do meio filtrante pela retenção de sólidos da amostra, mas sem a colmatação total (entupimento) do filtro. Isto indica que as eficiências de remoção podem ser melhoradas com o aumento do tempo de utilização do filtro.

Para isso utilizaram-se as configurações com melhor desempenho (PAC 10 e 12 mg. $\mathrm{L}^{-1}$ ) para realização de ensaios com um volume maior de amostra (6 litros). O aumento do volume filtrado visa a repetição do ensaio de coagulação/floculação (avaliação da repetibilidade do ensaio) e também uma maior retenção de sólidos em função do tempo de uso do meio filtrante, permitindo uma melhor eficiência do processo. Os resultados são apresentados na Figura 8.

Figura 8 - Filtração com dosagens de PAC $10 \mathrm{mg} . \mathrm{L}^{-1}$ e $12 \mathrm{mg} . \mathrm{L}^{-1}$ para o em pH natural 


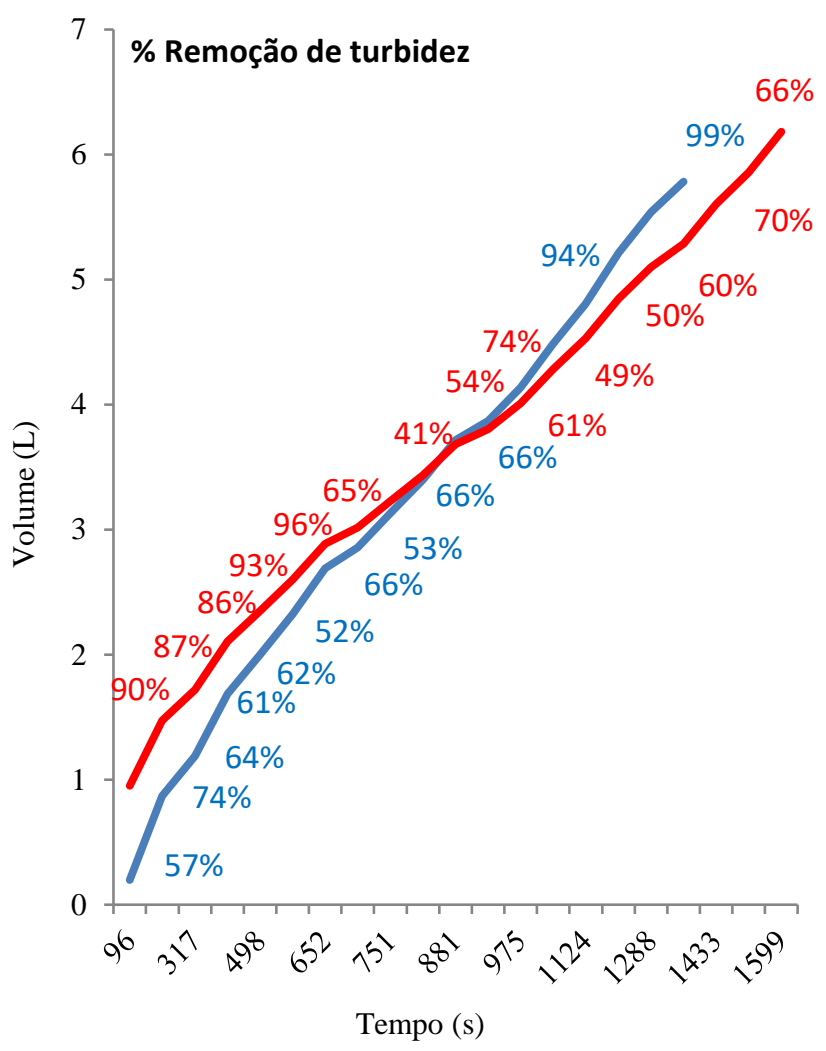

$\longrightarrow$ PAC 12 mg.L-1 —PAC 10 mg.L-1

PAC 12 mg.L $\mathrm{L}^{-1} \mathrm{R}^{2}=0,9914$; PAC $10 \mathrm{mg} \cdot \mathrm{L}^{-1} \mathrm{R}^{2}=0,9943$.

Com a utilização de um maior volume de amostra no processo de filtração foi possível avaliar melhor o desempenho do coagulante PAC, e verificouse que na dosagem de $12 \mathrm{mg} . \mathrm{L}^{-1}$ obteve-se maior eficiência na remoção de turbidez, atingindo $99 \%$ de remoção de turbidez, e com uma maior taxa filtração de $34 \mathrm{~m}^{3} \cdot \mathrm{m}^{-2} \cdot \mathrm{h}^{-1}$, enquanto que a dosagem de $10 \mathrm{mg} \cdot \mathrm{L}^{-}$ ${ }^{1}$ apresentou uma taxa de filtração de $27,5 \mathrm{~m}^{3} \cdot \mathrm{m}^{-2} \cdot \mathrm{h}^{-1}$. Como houve uma variação na eficiência de remoção de turbidez durante o período de filtração, o valor utilizado para uma comparação mais adequada das dosagens se deu pelo volume total do filtrado após o término da filtração.

Ao final de todos os ensaios de filtração foram realizados sete parâmetros para caracterização das amostras tratadas, sendo eles: turbidez, cor aparente, alumínio total, ferro total, condutividade, sólidos dissolvidos totais (SDT) e $\mathrm{pH}$, todos do volume total da amostra tratada. Os resultados são apresentados na Tabela 4.
De acordo com a Tabela 4 verificou-se que para o parâmetro físico, cor aparente, as amostras apresentaram diferenças mais significativas, que podem ser justificadas pela presença de ferro residual. $\mathrm{O}$ ferro no estado ferroso $\left(\mathrm{Fe}^{2+}\right)$ é oxidado para $\mathrm{Fe}^{3+}$ em virtude do oxigênio dissolvido na água, originando hidróxido de ferro insolúvel, conferindo cor e também turbidez para água.

Segundo CPRM (1997) para o ser humano a ausência de ferro causa anemia, assim como seu excesso aumenta a incidência de diabetes e problemas cardíacos.

\section{Conforme Portaria $\mathrm{n}^{\circ} \quad 2.914 / 2011$ do} Ministério da Saúde (BRASIL, 2011) em todas as dosagens utilizadas o padrão estabelecido foi cumprido, no entanto, a dosagem com $12 \mathrm{mg} . \mathrm{L}^{-1}$ de PAC demonstrou melhores eficiência em todos os parâmetros avaliados e com uma maior taxa de 
filtração, permitindo o tratamento de um maior volume de água em um menor tempo.

\section{Avaliação de viabilidade econômica dos coagulantes}

A viabilidade econômica dos coagulantes foi avaliada a partir dos seus valores comerciais e o volume de água tratada pela ETA, a Tabela 5 apresenta este estudo de custos.
Tabela 5 - Avaliação de custos

\begin{tabular}{c|c|c|c}
\hline Coagulante & $\begin{array}{c}\text { Preço } \\
\mathrm{R} \$ . \mathrm{L}^{-1}\end{array}$ & Volume/dia & $\begin{array}{c}\text { Custo } \\
\mathrm{R} \$ / \text { dia }\end{array}$ \\
\hline SAF 15 mg. $\mathrm{L}^{-1}$ & $0,78^{*}$ & \multirow{2}{*}{$16.632 \mathrm{~m}^{3}$} & 145,29 \\
\cline { 1 - 2 } PAC $10 \mathrm{mg} . \mathrm{L}^{-1}$ & $1,36^{*}$ & & 180,95 \\
\hline
\end{tabular}

*Fonte: Sulfago 2018 e BRK Ambiental.

Tabela 4 - Parâmetros avaliados após ensaios de coagulação seguidos de filtração.

\begin{tabular}{|c|c|c|c|c|c|c|c|}
\hline Coagulante & $\begin{array}{c}\text { Turbidez } \\
\text { (NTU) }\end{array}$ & $\begin{array}{c}\text { Cor } \\
\text { Aparente } \\
\text { (uC) }\end{array}$ & $\begin{array}{c}\text { Alumínio } \\
\text { Total } \\
\left(\mathrm{mg}^{\left.-L^{-1}\right)}\right.\end{array}$ & $\begin{array}{c}\text { Ferro } \\
\text { Total } \\
\left(\mathrm{mg}^{\left.-\mathrm{L}^{-1}\right)}\right.\end{array}$ & $\begin{array}{l}\text { Condutividade } \\
\qquad\left(\mu \mathrm{Scm}^{-1}\right)\end{array}$ & $\begin{array}{c}\text { SDT } \\
\left(\mathrm{mg} \cdot \mathrm{L}^{-1}\right)\end{array}$ & $\mathrm{pH}$ \\
\hline PAC 8 mg.L - $^{-1}$ & 1,81 & 11 & 0,008 & 0,08 & 28 & 14 & 7,5 \\
\hline PAC $10 \mathrm{mg} . \mathrm{L}^{-1} *$ & 1,36 & 4 & 0,015 & 0,02 & 29 & 15 & 7,6 \\
\hline PAC $12 \mathrm{mg} . \mathrm{L}^{-1} *$ & 0,55 & 0 & 0,018 & 0,03 & 27 & 14 & 7,4 \\
\hline SAF $15 \mathrm{mg} . \mathrm{L}^{-1}$ & 1,83 & 7 & 0,021 & 0,12 & 23 & 12 & 7,3 \\
\hline VMP** & 5 & 15 & 0,2 & 0,3 & - & 1000 & Entre 6 e 9,5 \\
\hline
\end{tabular}

* Análises realizadas após a filtração de 6 litros de amostra.

** Valor máximo permitido conforme a Portaria nº 2.914/2011 do Ministério da Saúde.

o PAC. Na remoção de cor aparente, turbidez, alumínio total e ferro total, o PAC (12 mg. $\left.\mathrm{L}^{-1}\right)$, demonstrou melhor desempenho com maior taxa de

O estudo de custos foi realizado a partir da produção média de água tratada pela ETA, a qual é de $16.632 \mathrm{~m}^{3} \mathrm{dia}^{-1}$. Esta avaliação mostra que apesar do coagulante PAC apresentar um desempenho satisfatório com $10 \mathrm{mg} . \mathrm{L}^{-1}$, a utilização do coagulante SAF tem maior viabilidade econômica mesmo sendo consumido em dosagens maiores.

Vale ressaltar que considerando a maior taxa de filtração do PAC sua utilização seria indicada a esta ETA, caso a mesma necessitasse aumentar a sua produção de água sem ampliação estrutural ou aumento de carga horária de operação.

\section{CONCLUSÃO}

Ambos os coagulantes analisados obtiveram bons resultados para remoção de turbidez da água bruta, visto que o SAF em uma dosagem maior do que filtração. A eficiência do PAC foi evidenciada com relação ao SAF, mas a substituição não seria pertinente visando questões econômicas, e de armazenamento, pois o PAC deve ser armazenado em local específico. De modo geral, para os dois coagulantes testados, os melhores resultados quanto a remoção de turbidez foram em pH natural $(6,7)$ da água bruta, não sendo necessária a utilização de alcalinizantes. As taxas de filtração realizadas no laboratório não foram condizentes com as empregadas em escala real, porém podem ser utilizadas para comparação dos coagulantes.

\section{AGRADECIMENTO}

Agradecemos a empresa BRK Ambiental pelo apoio ao desenvolvimento deste estudo. O presente trabalho foi realizado com o apoio do Conselho Nacional de Desenvolvimento Científico e 
Tecnológico - CNPq - Brasil. O desenvolvimento desta pesquisa contou com benefícios do Programa de Bolsa Produtividade da UFT (Propesq/UFT).

Todos os autores declararam não haver qualquer potencial conflito de interesses referente a este artigo.

\section{REFERÊNCIAS}

APHA (1999) Standard Methods for the Examination of Water and Wastewater. American Public Health Association, Washington DC.

BRASIL. Ministério da Saúde. Portaria 2.914, de 12 de dezembro de 2011. Dispõe sobre os procedimentos de controle e vigilância da qualidade da água para o consumo humano e seu padrão de potabilidade. Brasília: Ministério da Saúde, 2011.

CARDOSO, Patrícia Regina da Silva. Informações sobre o Policloreto de Alumínio. Resposta Técnica produzida pelo Serviço Brasileiro de Respostas Técnicas/SBRT06 de junho 2006. Disponível em < http://.sbrt.ibict.br> Acesso em 26/05/2018.

CONSTANTINO, Vera R. Leopoldo; ARAKI, Koiti; SILVA, Denise de Oliveira; OlLIVEIRA, Wanda de. Preparação de compostos de alumínio a partir da bauxita: considerações sobre alguns aspectos envolvidos em um experimento didático. Quím. Nova, vol.25, n.3, p.490-498, 2002.

DI BERNARDO, L.; DANTAS, A. D. B.; VOLTAN, P. E. N. Métodos e técnicas de tratamento de água. São Paulo: LDiBe, vol.3, 2017.

DI BERNARDO, Luiz. Métodos e técnicas de tratamento de água- Rio de Janeiro: ABES, v.2, 2005.

ECKENFELDER, W. Wesley. Industrial Water Pollution Control. 2 ed. McGraw-Hill (New York), 1989.
GONÇALVES, R.F.; PIOTTO, Z.C.; RESENDE, M.B. Influência dos Mecanismos de Coagulação da Água Bruta na Reciclagem de Coagulantes em Lodos de Estações de Tratamento de Água. in: $19^{\circ}$

CONGRESSO DE ENGENHARIA SANITÁRIA E AMBIENTAL. ABES - Associação Brasileira de Engenharia Sanitária e Ambiental. Foz do Iguaçu, 1997.

JAFELICCI JUNIOR, M; VARANDA, L.C. O Mundo dos Colóides. n. 9, p.11, maio 1999. Disponível em:<http://qnesc.sbq.org.br/online/qnesc09/quimsoc. pdf $>$. Acesso em: 14 de março de 2018.

OKUDA, T. et al. Removal of coagulant aluminum from water treatment residuals by acid. Water Research, New York, v. 60, p. 75-81, 2014.

PAVANELLI, G. (2001). Eficiência de diferentes tipos de coagulantes na coagulação, floculação e sedimentação de água com cor e turbidez elevada. São Carlos, 2001. 216p. Dissertação (Mestrado) - Escola de Engenharia São Carlos, Universidade de São Paulo.

PREFEITURA DE GURUPI. Prefeitura Municipal de Gurupi Disponível em: <http:www.gurupi.to.gov.br/?page=dadosmunicipio>. Acessado em 23 de abril de 2018.

RAMOS, I. R. O.; BRAGA, J. P. M.; ATAIIDE, J. V. A.; LIMA, A. P.; HOLANDA, L. Eletrostática em sistemas coloidais carregados. Revista Brasileira de Ensino de Física, vol. 40, nº 4, 2018.

RICHTER, C.A. (2009). Água: Métodos e Tecnologia de Tratamento. São Paulo: Editora Blucher, 2009. 340p.

SULFAGO - Sulfatos de Goiás LTDA. Disponível em: 〈http://www.sulfago.ind.br/site/>. Acessado em 02 de março de 2018. 